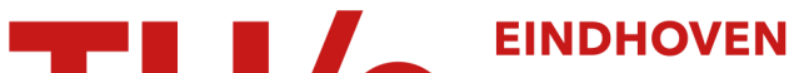 UNIVERSITY OF TECHNOLOGY
}

\section{On anomalous diffusion in a plasma in velocity space}

\section{Citation for published version (APA):}

Trigger, S. A., Ebeling, W., Heijst, van, G. J. F., Schram, P. P. J. M., \& Sokolov, M. (2010). On anomalous diffusion in a plasma in velocity space. Physics of Plasmas, 17(4), 042102-1/8.

https://doi.org/10.1063/1.3377779

DOI:

10.1063/1.3377779

Document status and date:

Published: 01/01/2010

\section{Document Version:}

Publisher's PDF, also known as Version of Record (includes final page, issue and volume numbers)

\section{Please check the document version of this publication:}

- A submitted manuscript is the version of the article upon submission and before peer-review. There can be important differences between the submitted version and the official published version of record. People interested in the research are advised to contact the author for the final version of the publication, or visit the DOI to the publisher's website.

- The final author version and the galley proof are versions of the publication after peer review.

- The final published version features the final layout of the paper including the volume, issue and page numbers.

Link to publication

\section{General rights}

Copyright and moral rights for the publications made accessible in the public portal are retained by the authors and/or other copyright owners and it is a condition of accessing publications that users recognise and abide by the legal requirements associated with these rights.

- Users may download and print one copy of any publication from the public portal for the purpose of private study or research.

- You may not further distribute the material or use it for any profit-making activity or commercial gain

- You may freely distribute the URL identifying the publication in the public portal.

If the publication is distributed under the terms of Article 25fa of the Dutch Copyright Act, indicated by the "Taverne" license above, please follow below link for the End User Agreement:

www.tue.nl/taverne

Take down policy

If you believe that this document breaches copyright please contact us at:

openaccess@tue.nl

providing details and we will investigate your claim. 


\title{
On anomalous diffusion in a plasma in velocity space
}

\author{
S. A. Trigger, ${ }^{1, a)}$ W. Ebeling, ${ }^{2}$ G. J. F. van Heijst, ${ }^{3}$ P. P. J. M. Schram, ${ }^{3}$ and I. M. Sokolov ${ }^{2}$ \\ ${ }^{1}$ Joint Institute for High Temperatures, Russian Academy of Sciences, Izhorskaya 13/19, \\ 125412 Moscow, Russia \\ ${ }^{2}$ Institut für Physik, Humboldt-Universität zu Berlin, Newtonstraße 15, D-12489 Berlin, Germany \\ ${ }^{3}$ Eindhoven University of Technology, P.O. Box 513, MB 5600 Eindhoven, The Netherlands
}

(Received 15 February 2010; accepted 10 March 2010; published online 12 April 2010)

\begin{abstract}
The problem of anomalous diffusion in momentum space is considered for plasmalike systems on the basis of a new collision integral, which is appropriate for consideration of the probability transition function (PTF) with long tails in momentum space. The generalized Fokker-Planck equation for description of diffusion (in momentum space) of particles (ions, grains, etc.) in a stochastic system of light particles (electrons or electrons and ions, respectively) is applied to the evolution of the momentum particle distribution in a plasma. In a plasma the developed approach is also applicable to the diffusion of particles with an arbitrary mass relation due to the small characteristic momentum transfer. The cases of an exponentially decreasing (including a Boltzmann-like) kernel in the PTF in momentum space, as well as more general kernels, which create anomalous diffusion in velocity space due to the long tail in the PTF, are considered. Effective friction and diffusion coefficients for plasmalike systems are found. (c) 2010 American Institute of Physics. [doi:10.1063/1.3377779]
\end{abstract}

\section{INTRODUCTION}

Diffusion in coordinate and in momentum (velocity) space is of fundamental importance and has attracted a growing interest during many years since the description of this process provides a simplified and effective key for addressing of many problems of the kinetic theory.

Deviations from a linear time dependence, i.e., $\left\langle r^{2}(t)\right\rangle \sim t$, of the mean square displacement in coordinate space have been experimentally observed, in particular, under essentially nonequilibrium conditions or for some disordered systems. The average square separation of a pair of particles passively moving in a turbulent flow grows, according to Richardson's law, with the third power of time. ${ }^{1}$ For diffusion typical for glasses and related complex systems, ${ }^{2}$ the observed time dependence is slower than linear. These two types of anomalous diffusion are obviously characterized as superdiffusion $\left\langle r^{2}(t)\right\rangle \sim t^{\alpha}(\alpha>1)$ and subdiffusion $(\alpha<1){ }^{3}$ For a description of these two diffusion regimes a number of effective models and methods have been suggested. The continuous time random walk model of Scher and Montroll, ${ }^{4}$ leading to subdiffusion behavior, provides a basis for understanding photoconductivity in strongly disordered and glassy semiconductors. The Levy-flight model, ${ }^{5}$ leading to superdiffusion, describes various phenomena as self-diffusion in micelle systems, ${ }^{6}$ reaction, and transport in polymer systems, ${ }^{7}$ and is applicable even to the stochastic description of financial market indices. ${ }^{8}$ Both cases can be effectively described by generalized diffusion equations with fractional derivatives in time or in coordinates, respectively. ${ }^{9}$ For example, different aspects of the anomalous diffusion in coordinate space were considered within this scheme in Refs. 10 and 11 .

\footnotetext{
${ }^{a)}$ Electronic mail: satron@mail.ru.
}

However, recently a more general approach has been suggested in Refs. 12 and 13. This approach allows us to reproduce the results of the standard fractional differentiation method in coordinate space, when the latter is applicable, and enable to describe more complicated cases of anomalous diffusion processes. In Ref. 14 this approach has also been applied to the case of diffusion in a time-dependent external field in coordinate space. In what follows, we concentrate on the problem of diffusion in momentum space in application to plasma systems.

Problems of diffusion in momentum space have been considered for plasmas in the fundamental study by Landau ${ }^{15}$ and later by Rostoker and Rosenbluth, Lenard, Balescu, Klimontovich, and many others. Various theoretical and experimental aspects of these investigations can be found in Refs. $16-18$.

Here our main interest is focused on anomalous diffusion in momentum space by using the methods developed in Refs. 12 and 13 for coordinate space. Recently, see Ref. 19, a new kinetic equation for anomalous diffusion in velocity space has been derived on the basis of an appropriate expansion of probability transition function (PTF) and some particular problems were investigated on this basis. In the present paper the problem of anomalous diffusion in momentum (velocity) space will be considered for plasmalike systems.

Some aspects of anomalous diffusion in velocity space have been investigated during the past decade in a number of studies. ${ }^{20-23}$ In particular, in Ref. 22 the phenomenological equation for anomalous diffusion in velocity space for a magnetized plasma has been obtained on the basis of the Langevin model with the linear friction proportional to the particle velocity and with non-Gauss noise. The corresponding Fokker-Planck-type equation included a diffusion term with a fractional derivative and the usual drift term with the 
first momentum derivative. The applicability of this equation is limited due to phenomenological nature of the considered model. We should mention the analogy of this equation with the structure of the equation for anomalous diffusion in coordinate space under action of an external field. ${ }^{11,14}$ An equation similar to that in Ref. 22 has recently been applied in Ref. 23 to describe the evolution of the velocity distribution function of strongly nonequilibrium hot and rarified plasmas. Such types of plasma exist, e.g., in tokomaks. On the whole, in comparison with anomalous diffusion in coordinate space, anomalous diffusion in velocity space has been studied to a modest extent.

In this paper the problem of anomalous diffusion in momentum (velocity) space will be considered for plasmalike systems. In spite of formal similarity, the physical (and mathematical) nature of diffusion in momentum space is very different from that in coordinate space. This is clear already from the fact that momentum conservation, which takes place in momentum space, has no analogy in coordinate space.

Diffusion in velocity space for the cases of normal and anomalous behavior of the PTF is presented in Sec. II. Starting from the argumentation based on the Boltzmann-type of the PTF, we describe the new approach to the kinetic equation, which in fact can be applied to the wide class of PTF functions based on the prescribed distribution function for one (light) sort of particles. The anomalous diffusion in momentum space for plasma is analyzed in Sec. III on the basis of the Boltzmann-type kernel for PTF. Models of anomalous diffusion for plasmalike systems are considered in Sec. IV. In Sec. V the generalized Fokker-Planck equation for diffusion, written for the formal Fourier component $f(\mathbf{s}, t)$ of the distribution function $f(\mathbf{p}, t)$, is represented in partial derivatives in velocity space. This representation is possible only in particular cases of the power dependence of the coefficients in the generalized diffusion equation.

\section{CALCULATION OF THE DIFFUSION IN THE VELOCITY SPACE ON THE BASIS OF A MASTER-TYPE EQUATION}

Let us consider now the main problem formulated in the introduction, namely, diffusion in momentum space ( $V$-space) on the basis of the master equation, which describes the balance of grains entering and leaving point $\mathbf{p}$ at time $t$ (see, e.g., Refs. 24 and 25),

$$
\frac{d f(\mathbf{p}, t)}{d t}=\int d \mathbf{p}\left\{w\left(\mathbf{p}, \mathbf{p}^{\prime}\right) f\left(\mathbf{p}^{\prime}, t\right)-w\left(\mathbf{p}^{\prime}, \mathbf{p}\right) f(\mathbf{p}, t)\right\} .
$$

The structure of this equation is formally similar to the master equation (see, e.g., Ref. 13) in coordinate space. Here $w\left(\mathbf{p}, \mathbf{p}^{\prime}\right)$ is the kernel describing the transition probabilities. Note that there is only one rather general condition, which $w\left(\mathbf{p}, \mathbf{p}^{\prime}\right)$ should satisfy if the stationary solution exists: the detailed balance condition for a stationary distribution function $f^{\text {st }}(\mathbf{p})$, which reads

$$
\frac{w\left(\mathbf{p}, \mathbf{p}^{\prime}\right)}{w\left(\mathbf{p}^{\prime}, \mathbf{p}\right)}=\frac{f^{\mathrm{st}}(\mathbf{p})}{f^{\mathrm{st}}\left(\mathbf{p}^{\prime}\right)} .
$$

In the following analysis we use a form of the master equation $^{26}$ equivalent to Eq. (1),

$$
\frac{d f(\mathbf{p}, t)}{d t}=\int d \mathbf{q}\{W(\mathbf{q}, \mathbf{p}+\mathbf{q}) f(\mathbf{p}+\mathbf{q}, t)-W(\mathbf{q}, \mathbf{p}) f(\mathbf{p}, t)\} .
$$

The probability transition $W\left(\mathbf{p}, \mathbf{p}^{\prime}\right)$ describes the probability for a grain with momentum $\mathbf{p}^{\prime}$ at point $\mathbf{p}^{\prime}$ in momentum space to transfer from this point $\mathbf{p}^{\prime}$ to the point $\mathbf{p}$ per unit time. The momentum transfer is equal to $\mathbf{q}=\mathbf{p}^{\prime}-\mathbf{p}$. Of course, as mentioned above, the overall momentum has to be conserved.

Assuming in the beginning that the characteristic changes in momentum are small, one may expand Eq. (3) and arrive at the Fokker-Planck form of the equation for the density distribution $f(\mathbf{p}, t)$,

$$
\begin{aligned}
& \frac{d f(\mathbf{p}, t)}{d t}=\frac{\partial}{\partial p_{\alpha}}\left\{A_{\alpha}(\mathbf{p}) f(\mathbf{p}, t)+\frac{\partial}{\partial p_{\beta}}\left[B_{\alpha \beta}(\mathbf{p}) f(\mathbf{p}, t)\right]\right\}, \\
& A_{\alpha}(\mathbf{p})=\int d^{r} q q_{\alpha} W(\mathbf{q}, \mathbf{p}), \\
& B_{\alpha \beta}(\mathbf{p})=\frac{1}{2} \int d^{r} q q_{\alpha} q_{\beta} W(\mathbf{q}, \mathbf{p}) .
\end{aligned}
$$

The coefficients $A_{\alpha}$ and $B_{\alpha \beta}$ describe the friction force and diffusion, respectively. Here $r$ is the momentum space dimension.

Because the velocity of heavy particles is small, the p-dependence of the PTF can be neglected for the calculation of diffusion coefficient, which in this case is constant $B_{\alpha \beta}=\delta_{\alpha \beta} B$, where $B$ is the integral

$$
B=\frac{1}{2 r} \int d^{r} q q^{2} W(q) .
$$

If we neglect the $\mathbf{p}$-dependence of the PTF at all, we arrive at $A_{\alpha}=0$ (while the diffusion coefficient is constant). In this approach, which is known to be incorrect, the coefficient $A_{\alpha}$ for the Fokker-Planck equation can be determined on the basis of the argument that the stationary distribution function is Maxwellian. In this way we arrive at the standard form of the coefficient $M T A_{\alpha}(p)=p_{\alpha} B$, which is one of the forms of Einstein's relation. For systems far from equilibrium this argument is not acceptable.

Following Ref. 1 we now generalize the Fokker-Planck approach to find the coefficients of the kinetic equation, which are applicable also to slowly decreasing PTFs. We apply a more general approach, based on the difference in the velocities for light and heavy particles. For calculation of the function $A_{\alpha}$ we have to take into account that the function $W(\mathbf{q}, \mathbf{p})$ is scalar and depends on the variables $q, \mathbf{q} \cdot \mathbf{p}, p$. Expanding $W(\mathbf{q}, \mathbf{p})$ on $\mathbf{q} \cdot \mathbf{p}$ one arrives at the following approximate representation of the function $W(\mathbf{q}, \mathbf{p})$ : 


$$
W(\mathbf{q}, \mathbf{p}) \simeq W(q)+\tilde{W}^{\prime}(q)(\mathbf{q} \cdot \mathbf{p})+\frac{1}{2} \tilde{W}^{\prime \prime}(q)(\mathbf{q} \cdot \mathbf{p})^{2},
$$

where $\quad \tilde{W}^{\prime}(q) \equiv \partial W(q, \mathbf{q} \cdot \mathbf{p}) /\left.\partial(\mathbf{q p})\right|_{\mathbf{q} \cdot \mathbf{p}=0} \quad$ and $\quad \tilde{W}^{\prime \prime}(q)$ $\equiv \partial^{2} W(q, \mathbf{q} \cdot \mathbf{p}) /\left.\partial(\mathbf{q p})^{2}\right|_{\mathbf{q} \cdot \mathbf{p}=0}$.

Then, with the necessary accuracy, $A_{\alpha}$ equals

$$
\begin{aligned}
A_{\alpha}(\mathbf{p}) & =\int d^{r} q q_{\alpha} q_{\beta} p_{\beta} \tilde{W}^{\prime}(q) \\
& =p_{\alpha} \int d^{r} q q_{\alpha} q_{\alpha} \tilde{W}^{\prime}(q) \\
& =\frac{p_{\alpha}}{r} \int d^{r} q q^{2} \tilde{W}^{\prime}(q) .
\end{aligned}
$$

If the function $W(\mathbf{q}, \mathbf{p})$ satisfies the equality $\tilde{W}^{\prime}(q)$ $=W(q) / 2 M T$, we obtain the known Einstein relation

$$
M T A_{\alpha}(\mathbf{p})=p_{\alpha} B \text {. }
$$

Let us check this relation for Boltzmann-type collisions, which are described by the PTF $W(\mathbf{q}, \mathbf{p})=w_{B}(\mathbf{q}, \mathbf{p}),{ }^{13}$

$$
\begin{aligned}
w_{B}(\mathbf{q}, \mathbf{p})= & \frac{2 \pi}{\mu^{2} q} \int_{q / 2 \mu}^{\infty} d u u \frac{d \sigma}{d o}\left[\arccos \left(1-\frac{q^{2}}{2 \mu^{2} u^{2}}\right), u\right] \\
& \times f_{b}\left(u^{2}+v^{2}-\mathbf{q} \cdot \mathbf{v} / \mu\right),
\end{aligned}
$$

where $(\mathbf{p}=M \mathbf{v})$ and $d \sigma(\chi, u) / d o, \mu$, and $f_{b}$ are the differential scattering cross section, the mass, and distribution function for the light particles, respectively. In Eq. (10) we took into account the approximate equalities for the scattering of light and heavy particles $q^{2}=(\Delta \mathbf{p})^{2}=p^{\prime 2}(1-\cos \theta)$ and $\theta \simeq \chi$, where $p^{\prime}=\mu u$ is the momentum of the light particle before collision.

For the equilibrium Maxwellian distribution $f_{b}^{0}$ the equality $\tilde{W}^{\prime}(q)=W(q) / 2 M T$ is evident and we derive the usual Fokker-Planck equation in velocity space with constant diffusion and friction coefficients $D=B / M^{2}$ and friction $\beta=B / M T=D M / T$, respectively, which satisfy the Einstein relation.

For some nonequilibrium situations the PTF, as a function of the variable $q$, possesses a long tail. In this case we have to derive a generalization of the kinetic equation in the spirit of the analysis of the coordinate case ${ }^{13,14}$ because the diffusion and friction coefficients in the form of Eqs. (6) and (8) diverge for large $q$ if the functions have an asymptotic behavior $W(q) \sim 1 / q^{\alpha}$ with $\alpha \leq r+2$ and (or) $\tilde{W}^{\prime}(q) \sim 1 / q^{\beta}$ with $\beta \leq r+2$.

Inserting expansion (7) for $W(\mathbf{q}, \mathbf{p})$ in Eq. (3) (as an example, we choose $r=3$; the analysis for arbitrary $r$ runs in a similar way) we arrive at a new collision term in the kinetic equation, which can be considered as a generalization of the Fokker-Planck equation for anomalous diffusion in velocity space, ${ }^{19}$

$$
\frac{d f(\mathbf{s}, t)}{d t}=A(s) f(\mathbf{s})+B_{\alpha}(s) \frac{\partial f(\mathbf{s}, t)}{\partial \mathbf{s}_{\alpha}} .
$$

In fact, as shown in Ref. 19, in expansion (7) for $W(\mathbf{q}, \mathbf{p})$ we have to keep (with the necessary accuracy) only the terms linear in $\mathbf{q p}$ and $\mathbf{p}$. The function $f(\mathbf{s})$ in Eq. (11) is the
Fourier-component $f(\mathbf{s})=\int\left[d \mathbf{p} /(2 \pi)^{3}\right] \exp (i \mathbf{p s}) f_{g}(\mathbf{p}, t)$ and the coefficients are equal to

$$
\begin{aligned}
A(s) & =\int d \mathbf{q}\{\exp [-i(\mathbf{q s})]-1\} W(q) \\
& =4 \pi \int_{0}^{\infty} d q q^{2}\left[\frac{\sin (q s)}{q s}-1\right] W(q), \\
B_{\alpha} \equiv s_{\alpha} B(s), & \\
B(s) & =-\frac{i}{s^{2}} \int d \mathbf{q q s}\{\exp [-i(\mathrm{qs})-1]\} \tilde{W}^{\prime}(q) \\
& =\frac{4 \pi}{s^{2}} \int_{0}^{\infty} d q q^{2}\left[\cos (q s)-\frac{\sin (q s)}{q s}\right] \tilde{W}^{\prime}(q) .
\end{aligned}
$$

Here we took into account the existence of the small parameter $\mu / M$ and we omitted the small on this parameter terms of order $\mathbf{p}^{2}$ and $W^{\prime \prime}$ in Eq. (7).

For the isotropic function $f(\mathbf{s})=f(s)$ one can rewrite Eq. (11) in the form

$$
\frac{d f(s, t)}{d t}=A(s) f_{g}(s)+B(s) s \frac{\partial f(s, t)}{\partial s} .
$$

For the case of strongly decreasing PTF the exponent under the integrals for the functions $A(s)$ and $B(s)$ can be expanded as

$$
A(s) \simeq=-\frac{s^{2}}{6} \int d \mathbf{q} q^{2} W(q), \quad B(s) \simeq-\frac{1}{3} \int d \mathbf{q} q^{2} \tilde{W}^{\prime}(q) .
$$

Then the simplified kinetic equation for the case of short range on $q$-variable PTF (nonequilibrium, in general case) reads

$$
\frac{d f(s, t)}{d t}=A_{0} s^{2} f(s)+B_{0} s \frac{\partial f(s)}{\partial s},
$$

where $A_{0} \equiv-1 / 6 \int d \mathbf{q} q^{2} W(q)$ and $B_{0} \equiv-1 / 3 \int d \mathbf{q} q^{2} \tilde{W}^{\prime}(q)$.

The stationary solution of Eq. (14) reads

$$
f(s, t)=C \exp \left[-\int_{0}^{s} d s^{\prime} \frac{A\left(s^{\prime}\right)}{s^{\prime} B\left(s^{\prime}\right)}\right]=C \exp \left[-\frac{A_{0} s^{2}}{2 B_{0}}\right] \text {. }
$$

The corresponding normalized stationary momentum distribution is given by

$$
f(p)=\frac{N B_{0}^{3 / 2}}{\left(2 \pi A_{0}\right)^{3 / 2}} \exp \left[-\frac{B_{0} p^{2}}{2 A_{0}}\right] .
$$

Therefore, in Eq. (17) the constant $C=N$, where $N$ is the total number of particles in the system, which undergo diffusive motion. Equation (16) and this distribution are the generalization of the Fokker-Planck case for normal diffusion in a nonequilibrium situation with strongly decreasing kernels $W(q), W^{\prime}(q)$, when the prescribed PTF function $W(\mathbf{q}, \mathbf{p})$ is determined, e.g., by some non-Maxwellian distribution of the small particles $f_{b}$. To show this in an alternative way, let us 
take the Fourier transformation of Eq. (11) and the corresponding coefficients $A$ and $B_{\alpha}$,

$$
\frac{d f(\mathbf{p}, t)}{d t}=-A_{0} \frac{\partial^{2} f(\mathbf{p}, t)}{\partial p^{2}}-B_{0} \frac{\partial\left[p_{\alpha} f(\mathbf{p}, t)\right]}{\partial p_{\alpha}} .
$$

We then arrive at a Fokker-Planck-type equation with friction coefficient $\beta=-B_{0}$ and diffusion coefficient $D=-A_{0} / M^{2}$. In general these coefficients [Eq. (15)] do not satisfy the Einstein relation.

In the case of equilibrium $W$-function (e.g., $f_{b}=f_{b}^{0}$, see above) the equality $\tilde{W}^{\prime}(q)=W(q) / 2 M T_{b}$ is satisfied. Then we find $A(s) / s B(s) \equiv A_{0} / B_{0}=M T_{b}$. In this case the Einstein relation between the diffusion and friction coefficients $D$ $=\beta T / M$ is satisfied and the standard Fokker-Planck equation is valid. In the general case, however, the general equations (11)-(13) have to be used.

\section{DIFFUSION IN PLASMA SYSTEMS ON THE BASIS OF BOLTZMANN-TYPE COLLISIONS}

Let us calculate the PTF for the case of Coulomb collisions. The differential cross section for the Coulomb scattering $d \sigma / d o$ equals

$$
\begin{aligned}
\frac{d \sigma(\chi, u)}{d o} & =\left(Z e^{2} / 2 \mu u^{2}\right)^{2} \frac{1}{\sin ^{4} \frac{\chi}{2}} \\
& =\left(Z e^{2} / 2 \mu u^{2}\right)^{2} \frac{16 \mu^{4} u^{4}}{q^{4}},
\end{aligned}
$$

where $\chi=\arccos \left(1-q^{2} / 2 \mu^{2} u^{2}\right)$. Then

$$
\begin{aligned}
w_{B}^{\text {Coul }}(\mathbf{q}, \mathbf{p})= & \frac{2 \pi}{\mu^{2} q} \int_{q / 2 \mu}^{\infty} d u u\left(Z e^{2} / 2 \mu u^{2}\right)^{2} \\
& \times \frac{16 \mu^{4} u^{4}}{q^{4}} f_{b}\left(u^{2}+v^{2}-\mathbf{q} \cdot \mathbf{v} / \mu\right) \\
= & \frac{8 Z^{2} e^{4} \pi}{q^{5}} \int_{q / 2 \mu}^{\infty} d u u f_{b}\left(u^{2}+v^{2}-\mathbf{q} \cdot \mathbf{v} / \mu\right) .
\end{aligned}
$$

It is necessary to stress that in the case of the Coulomb interaction the general equations (11) and (14) are applicable not only for diffusion of heavy particles in a light particle medium but also for arbitrary mass relations. The reason for this statement is the typical small transfer of momenta in the Coulomb systems.

Let us calculate now the coefficients $A_{\alpha}(\mathbf{p})$ and $B_{\alpha \beta}(\mathbf{p})$ to compare the results with the linearized Landau kinetic equation, in which these coefficients depend on $\mathbf{p}$. This implies that for the Coulomb interaction the expansion by $(\mathbf{q} \cdot \mathbf{p})$ has to be performed at finite $\mathbf{p}$.

At first we consider the approximation in the spirit of the usual Fokker-Planck approach. Equations (5) and (8) lead to the expressions

$$
A_{\alpha}(\mathbf{p})=\int d^{3} q q_{\alpha} w_{B}^{\mathrm{Coul}}(\mathbf{q}, \mathbf{p}) \cong \frac{p_{\alpha}}{3} \int d^{3} q q^{2} \widetilde{w}_{B}^{\text {Coul }}(q),
$$

$$
\begin{aligned}
B_{\alpha \beta}(\mathbf{p}) & =\frac{1}{2} \int d^{3} q q_{\alpha} q_{\beta} w_{B}^{\mathrm{Coul}}(\mathbf{q}, \mathbf{p}) \\
& \cong \frac{1}{2} \int d^{3} q q_{\alpha} q_{\beta} w_{B}^{\mathrm{Coul}}(q),
\end{aligned}
$$

where

$$
\begin{aligned}
& w_{B}^{\text {Coul }}(q)=\frac{8 Z^{2} e^{4} \pi}{q^{5}} \int_{q / 2 \mu}^{\infty} d u u f_{b}\left(u^{2}\right), \\
& \widetilde{w}_{B}^{\prime \text { Coul }}(q)=-\frac{8 Z^{2} e^{4} \pi}{M \mu q^{5}} \int_{q / 2 \mu}^{\infty} d u u f_{b}^{\prime}\left(u^{2}\right), \\
& \widetilde{w}_{B}^{\prime \text { Coul }}(q)=-\frac{4 Z^{2} e^{4} \pi}{M T q^{5}} \int_{q / 2 \mu}^{\infty} d u u f_{b}\left(u^{2}\right),
\end{aligned}
$$

and $f_{b}^{\prime}(y) \equiv \partial f_{b}(y) / \partial y$. The procedure that we used here implies that the long tails of the functions $w_{B}^{\text {Coul }}(q)$ and $\widetilde{w}_{B}^{\prime C o u l}(q)$ are absent. It is easy to see that expressions (24) and (25) in the limit of small $q$ (the lower limit of the integrals in these equations is taken equal to zero, which corresponds to the Landau small- $q$ expansion) are appropriate in the Fokker-Planck equation to the Landau approach for the kinetic equation for plasma. In this case the coefficients $A_{\alpha}(\mathbf{p})$ and $B_{\alpha \beta}(\mathbf{p})$ read

$$
\begin{aligned}
& A_{\alpha}(\mathbf{p}) \cong \frac{p_{\alpha}}{3} \int d^{3} q q^{2} \widetilde{w}_{B}^{\prime \text { Coul }}(q) \\
& \cong-\frac{16 \pi^{2} Z^{2} e^{4} p_{\alpha}}{3 M T} \ln \left(\frac{q_{\max }}{q_{\min }}\right) J \\
& B_{\alpha \beta} \cong \frac{\delta_{\alpha \beta}}{6} \int d^{3} q q^{2} w_{B}^{\mathrm{Coul}}(q) \cong \delta_{\alpha \beta} \frac{16 \pi^{2} Z^{2} e^{4}}{3} \ln \left(\frac{q_{\max }}{q_{\min }}\right) J
\end{aligned}
$$

$$
J=\int_{0}^{\infty} d u u f_{b}\left(u^{2}\right)=\frac{n \sqrt{\mu}}{(2 \pi)^{3 / 2} \sqrt{T}} .
$$

Therefore, one can rewrite $A_{\alpha} \equiv-p_{\alpha} \nu_{i e}$, where $\nu_{i e}$ is the characteristic frequency friction ions on electrons,

$$
\nu_{i e}=\frac{4 \sqrt{2 \pi} Z^{2} e^{4} n \mu^{1 / 2}}{3 M T^{3 / 2}} \ln \left(\frac{q_{\max }}{q_{\min }}\right) .
$$

The corresponding friction force per unit volume $F_{i e}$ is equal $\mathbf{F}_{\mathbf{i e}}=n_{i} M \mathbf{U} \nu_{i e}$, where $\mathbf{U}$ is the relative velocity of the electrons and ions. ${ }^{27}$ In fact, the divergence at large $q$ handled by a cutoff is an artifact. This becomes clear when calculating the equilibrium function $w_{B}^{\mathrm{Coul}, 0}(\mathbf{q}, \mathbf{p})$ more accurately, without expansion on small values of $q$.

For the equilibrium distribution function $f_{b}^{0}(u)$ $=n_{e}(\mu / 2 \pi T)^{3 / 2} \exp \left(-\mu u^{2} / 2 T\right)$ the PTF function reads 


$$
\begin{aligned}
w_{B}^{\text {Coul }, 0}(\mathbf{q}, \mathbf{p})= & \frac{4 n_{e} Z^{2} e^{4} \pi}{q^{5}} \\
& \times \exp \left[-\mu\left(v^{2}-\mathbf{q} \cdot \mathbf{v} / \mu\right) / 2 T\right] \\
& \times \int_{q / 2 \mu}^{\infty} d u^{2}(\mu / 2 \pi T)^{3 / 2} \exp \left[-\mu u^{2} / 2 T\right] \\
= & \frac{4 n_{e} Z^{2} e^{4} \mu^{1 / 2}}{\sqrt{2 \pi T} q^{5}} \\
& \times \exp \left[-\mu\left(v^{2}-\mathbf{q} \cdot \mathbf{v} / \mu+q^{2} / 4 \mu^{2}\right) / 2 T\right],
\end{aligned}
$$

$$
\begin{aligned}
w_{B}^{\prime \text { Coul }, 0}(\mathbf{q}, \mathbf{p})= & \frac{2 n_{e} Z^{2} e^{4} \mu^{1 / 2}}{\sqrt{2 \pi M T^{3}} q^{5}} \\
& \times \exp \left[-\mu\left(v^{2}-\mathbf{q} \cdot \mathbf{v} / \mu+q^{2} / 4 \mu^{2}\right) / 2 T\right] .
\end{aligned}
$$

Using the Fokker-Planck approximation for the coefficients $A_{\alpha}(\mathbf{p})$ and $B_{\alpha \beta}(\mathbf{p})$, and Eqs. (35) and (31) we find

$$
\begin{aligned}
& A_{\alpha}(\mathbf{p}) \cong \frac{p_{\alpha}}{3} \int d^{3} q q^{2} \widetilde{w}_{B}^{\prime \text { Coul }, 0}(\mathbf{q}, \mathbf{0})=\frac{p_{\alpha} 2 n_{e} Z^{2} e^{4} \mu^{1 / 2}}{3 \sqrt{2 \pi M T^{3}}} J_{1}, \\
& B_{\alpha \beta} \cong \frac{\delta_{\alpha \beta}}{6} \int d^{3} q q^{2} w_{B}^{\mathrm{Coul}, 0}(\mathbf{q}, \mathbf{0})=\frac{2 n_{e} Z^{2} e^{4} \mu^{1 / 2} \delta_{\alpha \beta}}{3 \sqrt{2 \pi T}} J_{1},
\end{aligned}
$$

where

$$
\begin{aligned}
J_{1} & =\int d^{3} q q^{-3} \exp \left[-q^{2} / 8 \mu T\right] \\
& =4 \pi \int_{0}^{\infty} \frac{d q}{q} \exp \left[-q^{2} / 8 \mu T\right] \\
& \simeq 4 \pi \int_{q_{\min }}^{\infty} \frac{d q}{q} \exp \left[-q^{2} / 8 \mu T\right] \\
& =2 \pi \int_{q_{\min }^{2} / 8 \mu T}^{\infty} \frac{d \zeta}{\zeta} \exp [-\zeta]=-\left.2 \pi E i\left(-q^{2} / 2 \mu T\right)\right|_{q_{\min }} ^{\infty} \\
& \simeq-2 \pi E i\left(-q_{\min }^{2} / 2 \mu T\right) .
\end{aligned}
$$

We can suppose that the minimal momentum transfer $q_{\min }$ is determined from the equality $q_{\min }^{2} / 2 \mu T=r_{\min } / r_{\max }$. According to the Landau theory for a weakly interacting plasma $r_{\min } / r_{\max }=Z e^{2} / T r_{D} \ll 1$ for $Z e^{2} / \hbar v_{T} \gg 1$, or $r_{\min } / r_{\max }$ $=\hbar^{2} / 2 \mu T r_{D} \ll 1$ for the opposite inequality $Z e^{2} / \hbar v_{T} \ll 1$.

Here $r_{D}$ is the Debye radius and $v_{T}=\sqrt{T / \mu}$ is of the order of the thermal velocity. In our approach the cutoff for the small momenta is satisfied automatically and corresponds to the second inequality (the "quantum" case).

For a weakly nonideal plasma this means a cutoff at the minimal momentum $q_{\min }=\hbar / r_{D}$. Then

$$
J_{1}=-2 \pi C+4 \pi \ln \left(\frac{r_{D}}{r_{\min }}\right) \simeq 4 \pi \ln \left(\frac{r_{D}}{r_{\min }}\right),
$$

where $r_{\min }^{2} \equiv \hbar^{2} / 2 \mu T$ and $C \simeq 0.577$ is the Euler constant.

It is easy to verify that $\tilde{W}^{\prime}(q)=W(q) / 2 M T$ and $\tilde{W}^{\prime \prime}(q)$ $=W(q) / 4 M^{2} T^{2}$ [in the case under consideration $W(\mathbf{q}, \mathbf{p})$ $\left.\equiv w_{B}^{\text {Coul }, 0}(\mathbf{q}, \mathbf{p})\right]$. Therefore, for the equilibrium case the usual Fokker-Planck equation for heavy particles (ions or dusty particles in dusty plasmas) is, naturally, valid with a good accuracy, owing to the exponential convergence of the integrals in the coefficients $A(s)$ and $B_{\alpha}(s)$ at high values of $q$. The term with $W^{\prime \prime}$ in Eq. (7) is negligible, according to the above general statement. However, for small $q$ the coefficients $A$ and $B_{\alpha}$ have the logarithmical divergence typical for the Coulomb interaction because $W \sim 1 / q^{5}$, just like $W^{\prime}(q)$. As follows from Eq. (21), this divergence not only exists for equilibrium but for an arbitrary distribution function $f_{b}$. The simplest physical way to avoid this divergence is to cut the integrals for $A$ and $B_{\alpha}$ in Eqs. (12) and (13) for small $q$ by the Debye radius $1 / r_{D}$, following the well known Landau procedure. We are more interested to find examples for nonexponential behavior of $W$, which may occur, e.g., for some specific non-Maxwellian distributions $f_{b}$.

\section{MODELS OF ANOMALOUS DIFFUSION FOR COULOMB INTERACTION}

Now we can calculate the coefficients for models of anomalous diffusion in plasmalike systems. At first we calculate the model of a Coulomb system with two species of particles with masses $\mu$ and $M \gg \mu$. Let us suppose that in the model under consideration the small particles are described by a prescribed stationary distribution $f_{b}=n_{b} \phi_{b} / u_{0}^{3}$ (where $\phi_{b}$ is the dimensionless distribution function and $u_{0}$ is the characteristic velocity for the distribution of the small particles) and $\xi \equiv\left(u^{2}+v^{2}-\mathbf{q} \cdot \mathbf{v} / \mu\right) / u_{0}^{2}$,

$$
W_{a}(\mathbf{q}, \mathbf{p})=\frac{8 \pi Z^{2} e^{4} n_{b}}{u_{0} q^{5}} \int_{\left(q^{2} / 4 \mu^{2}+v^{2}-\mathbf{q} \cdot \mathbf{v} / \mu\right) / u_{0}^{2}}^{\infty} d \xi \cdot \phi_{b}(\xi) .
$$

First, let us consider a power-law distribution $\phi_{b}(\xi)$ $=C / \xi^{\gamma}(\gamma>1)$,

$$
W_{a}(\mathbf{q}, \mathbf{p})=\left.\frac{8 \pi Z^{2} e^{4} n_{b} C}{u_{0} q^{5}} \frac{\xi^{1-\gamma}}{(1-\gamma)}\right|_{\xi 0} ^{\infty}=\frac{8 \pi Z^{2} e^{4} n_{b} C}{u_{0} q^{5}} \frac{\xi_{0}^{1-\gamma}}{\gamma-1},
$$

where $\xi_{0} \equiv\left(q^{2} / 4 \mu^{2}+v^{2}-\mathbf{q} \cdot \mathbf{v} / \mu\right) / u_{0}^{2}$.

For the case $p=0$ the value $\xi_{0} \rightarrow \widetilde{\xi}_{0} \equiv q^{2} / 4 \mu^{2} u_{0}^{2}$ and we arrive at the following expression for anomalous $W \equiv W_{a}$ :

$$
\begin{aligned}
W_{a}(\mathbf{q}, \mathbf{p}=\mathbf{0}) & =\frac{8 \pi Z^{2} e^{4} n_{b} C}{u_{0} q^{5}} \frac{\left(q / 2 \mu u_{0}\right)^{2-2 \gamma}}{\gamma-1} \\
& =\frac{2^{2 \gamma+1} \pi Z^{2} e^{4} n_{b} u_{0}^{2 \gamma-3} \mu^{2 \gamma-2} C}{(\gamma-1) q^{2 \gamma+3}} .
\end{aligned}
$$

To determine the structure of the transport process and the kinetic equation in velocity space we have to determine also the functions $\widetilde{W}^{\prime}(q)$ and $\widetilde{W}^{\prime \prime}(q)$. 
If $p \neq 0$ we have to use the full expression $\xi_{0}$ $\equiv\left(q^{2} / 4 \mu^{2}+p^{2} / M^{2}-\mathbf{q} \cdot \mathbf{p} / M \mu\right) / u_{0}^{2}$ and its derivatives on $\mathbf{q} \cdot \mathbf{p}$ at $p=0: \xi_{0}^{\prime}=-1 / M \mu u_{0}^{2}$ and $\xi_{0}^{\prime \prime}=0$. Then

$$
\tilde{W}^{\prime}(\mathbf{q}, \mathbf{p}) \equiv \frac{8 \pi Z^{2} e^{4} n_{b} C}{M \mu u_{0}^{3} q^{5} \xi_{0}^{\gamma}}, \quad \tilde{W}^{\prime \prime}(\mathbf{q}, \mathbf{p}) \equiv \frac{8 \pi \gamma Z^{2} e^{4} n_{b} C}{M^{2} \mu^{2} u_{0}^{5} q^{5} \xi_{0}^{\gamma+1}} .
$$

For $p=0\left(\xi_{0} \rightarrow \tilde{\xi}_{0}\right)$ we obtain the functions

$$
\begin{aligned}
& \widetilde{W}^{\prime}(q) \equiv \frac{2^{2 \gamma+3} \mu^{2 \gamma-1} u_{0}^{2 \gamma-3} \pi Z^{2} e^{4} n_{b} C}{M q^{2 \gamma+5}}, \\
& \widetilde{W}^{\prime \prime}(q) \equiv \frac{2^{2 \gamma+5} \gamma \mu^{2 \gamma} u_{0}^{2 \gamma-3} \pi Z^{2} e^{4} n_{b} C}{M^{2} q^{2 \gamma+7}} .
\end{aligned}
$$

For the function $A(s)$, according to Eq. (12), we find

$$
\begin{aligned}
A(s) & \equiv 4 \pi \int_{0}^{\infty} d q q^{2}\left[\frac{\sin (q s)}{q s}-1\right] W(q) \\
& =4 \pi C_{a} \int_{0}^{\infty} d q \frac{1}{q^{2 \gamma+1}}\left[\frac{\sin (q s)}{q s}-1\right] .
\end{aligned}
$$

Comparing the reduced equation (see below) in velocity space with the diffusion in coordinate space $[2 \gamma+3 \leftrightarrow \alpha$ and $\left.W(q)=C_{a} / q^{2 \gamma+3}\right]$ we can conclude that the integral in the right-hand side of Eq. (41) ( $3 d$ case) converges if $3<2 \gamma+3<5$ or $0<\gamma<1$. The inequality $\gamma<1$ implies convergence for small $q(q \rightarrow 0)$ and the inequality $\gamma>0$ implies convergence for $q \rightarrow \infty$. Likewise for the integral in $B(s)$,

$$
B(s)=\frac{4 \pi}{s^{2}} \int_{0}^{\infty} d q q^{2}\left[\cos (q s)-\frac{\sin (q s)}{q s}\right] \tilde{W}^{\prime}(q),
$$

convergence of $B(s)$ exists for small $q$ if $\gamma<0$ and for large $q \rightarrow \infty$ if $\gamma>-3 / 2$. Again, it is easy to show that the term with $W^{\prime \prime}$ can be omitted.

Therefore, for convergence of $A$ and $B$ for a large $q$ we require convergence for $A$, which implies $\gamma>0$. For convergence for small $q$ it is sufficient to have convergence for $B$, implying $\gamma<0$. Therefore, for the case of pure power-law behavior of the function $f_{b}(\xi)$ convergence is absent. It is also clear that the function $f_{b}(\xi)=C / \xi^{\gamma}(\gamma>1)$ cannot be normalized. However, for anomalous diffusion in momentum space in reality the convergence for small $q$ is always obtained, e.g., by a finite value of $v$ or by a change in the small $q$-behavior of $W(q)$ by screening (compare with the examples of anomalous diffusion in coordinate space ${ }^{13}$ ). Therefore, in the model under consideration, the "anomalous diffusion in velocity space" for a power-law behavior $f_{b}(u)$ [and as a consequence with a power-law dependence of $W(q)$ and $\left.\tilde{W}^{\prime}(q)\right]$ on large $q$ exists if for large $q$ the asymptotic behavior of $W(q \rightarrow \infty) \sim 1 / q^{2 \gamma+3}$ with $\gamma>0$. At the same time the expansion of the exponential function in Eqs. (12) and (13) under the integrals, leading to a Fokker-Planck-type kinetic equation, is invalid for power-type kernels $W(\mathbf{q}, \mathbf{p})$.

As an example of the above statements, let us consider the Cauchy-Lorentz-like distribution for the function $f_{b}$ $(r=3)$,

$$
f_{b}\left(u^{2}\right)=n_{b} \frac{v_{0}}{\pi^{2}\left(u^{2}+v_{0}^{2}\right)^{2}} .
$$

Then we find

$$
\begin{aligned}
W_{a}(\mathbf{q}, \mathbf{p}) & =\frac{8 Z^{2} e^{4} \pi}{q^{5}} \int_{q / 2 \mu}^{\infty} d u u f_{b}\left(u^{2}+v^{2}-\mathbf{q} \cdot \mathbf{v} / \mu\right) \\
& =\frac{4 v_{0}^{3} Z^{2} e^{4}}{\pi q^{5}} \int_{\xi_{0}}^{\infty} d \xi \frac{1}{1+\xi^{2}} \\
& =\frac{4 v_{0}^{3} Z^{2} e^{4}}{\pi q^{5}}\left\{\frac{\pi}{2}-\arctan \xi_{0}\right\},
\end{aligned}
$$

where $\xi_{0} \equiv\left(q^{2} / 4 \mu^{2}+v^{2}-\mathbf{q} \cdot \mathbf{v} / \mu\right) / v_{0}^{2}$ and

$$
\widetilde{W}_{a}^{\prime}(\mathbf{q}, \mathbf{p})=\frac{4 v_{0} Z^{2} e^{4}}{M \mu \pi q^{5}} \frac{1}{\xi_{0}^{2}+1} .
$$

For large $q$ functions (44) and (45) tend to $W_{a}(\mathbf{q}, \mathbf{p})$ $\simeq 16 v_{0}^{5} \mu^{2} Z^{2} e^{4} / \pi q^{7}$ and $\tilde{W}_{a}^{\prime}(\mathbf{q}, \mathbf{p}) \simeq 64 v_{0}^{5} \mu^{3} Z^{2} e^{4} / M \pi q^{9}$. For small $q$ convergence of the coefficients $A(s)$ and $B(s)$ cannot be obtained since these functions are determined by the expressions $W_{a}(\mathbf{q}, 0)$ and $\widetilde{W}_{a}^{\prime}(\mathbf{q}, 0)$. However, this problem can be avoided by using a cutoff of the respective integrals (41) and (42) at small $q$ or by modification of the distribution (43) at small $q$ (in the spirit of the respective cutoff for anomalous diffusion in coordinate space ${ }^{12}$ ). For large $q$ the CauchyLorentz-type of distributions have long tails, thus leading to anomalous diffusion.

Let us now consider the formal general model for which we will not connect the functions $W(q)$ and $\tilde{W}^{\prime}(q)$ with a concrete form of $W(\mathbf{q}, \mathbf{p})$. Therefore we consider the problem suggesting some behavior of the function $W(\mathbf{q}, \mathbf{p})$ but not on the level of the distribution function $f_{a}$. In general the functional $W(\mathbf{q}, \mathbf{p})$ is unknown. In this case one can suggest that independently one from another, the functions $W(q)$, $\tilde{W}^{\prime}(q)$, and $\tilde{W}^{\prime \prime}(q)$ possess a power-type $q$-dependence for a large $q$.

As an example, this dependence can be taken as the power type for two functions $W(q) \equiv a / q^{a}$ and $\tilde{W}^{\prime}(q)$ $\equiv b / q^{\beta}$, where $\alpha>0$ and $\beta$ are independent. Then, as follows from the consideration above, convergence of the function $W$ exists if $5>\alpha>3$ (for asymptotically small and large $q$, respectively). For the function $\tilde{W}^{\prime}(q)$ the convergence condition is $5>\beta>2$ for asymptotically small and large $q$, respectively.

Finally for the function $\widetilde{W}^{\prime \prime}(q)$ the convergence condition is $7>\eta>5$ (for asymptotically small and large $q$, respectively). In this case the terms with $W^{\prime \prime}$ can be omitted (for the same reasons as above).

For this example the kinetic equation (11) reads

$$
\frac{d f(\mathbf{s}, t)}{d t}=P_{0} s^{\alpha-3} f(\mathbf{s}, t)+s^{\beta-5} P_{1} s_{i} \frac{\partial}{\partial s_{i}} f(\mathbf{s}, t),
$$

where

$$
P_{0}=4 \pi a \int_{0}^{\infty} d \zeta \zeta^{2-\alpha}\left[\frac{\sin \zeta}{\zeta}-1\right],
$$




$$
P_{1}=4 \pi b \int_{0}^{\infty} d \zeta \zeta^{2-\beta}\left[\cos \zeta-\frac{\sin \zeta}{\zeta}\right]
$$

Taking into account the isotropy in $s$-space we can rewrite Eq. (46) in the form

$$
\frac{d f(s, t)}{d t}=P_{0} s^{\alpha-3} f(s, t)+s^{\beta-4} P_{1} \frac{\partial}{\partial s} f(s, t),
$$

Naturally, Eqs. (46) and (49) can be formally rewritten in momentum (or in velocity) space via the fractional derivatives of various orders (see below). Therefore, as is easy to see, for the purely power-law behavior of the functions $W(q)$ and $\tilde{W}^{\prime}(q)$ the solution with the convergent coefficients exists for powers in the intervals mentioned above. We establish that the universal type of anomalous diffusion in velocity space in the case under consideration exists if $5>\alpha>3$ or $5>\beta>2$. This universality seems similar to the universality of the Levy distribution in coordinate space, where the power $\alpha$ of the dependence of the PTF in coordinate space, $W(\widetilde{q}) \sim C / \widetilde{q}^{\alpha}$ on the displacement $\widetilde{q}$ lies in the interval 0 $<\mu=\alpha-r<2$ ( $r$ is the dimension of the coordinate space).

As is easy to see the stationary solution of Eq. (49) reads

$$
f(s)=C^{\prime} \exp \left[-\frac{P_{0} s^{\alpha-\beta+2}}{(\alpha-\beta+2) P_{1}}\right] .
$$

Of course, the general description above is also valid for the more complicated functions $W$ and $\tilde{W}^{\prime}$, possessing a nonpower dependence on $q$ at small $q$ and an asymptotical power dependence on $q$ at large $q$. In this case the limitations for convergence are connected only with large values of $q$, namely, it is enough to provide the inequalities $\alpha>3$ and $\beta>2$. Simple examples of such type of PTFs are (in analogy with anomalous diffusion in coordinate space ${ }^{13}$ )

$$
W_{a}(\mathbf{q})=\frac{1-\exp \left(-\gamma q^{n}\right)}{q^{\alpha}}, \quad \tilde{W}_{a}^{\prime}(\mathbf{q})=\frac{1-\exp \left(-\delta q^{m}\right)}{q^{\beta}} .
$$

The corresponding kinetic equations in these cases cannot be written in partial derivatives and evolution of the system has to be described by Eq. (11), or for the isotropic case by Eq. (14). If external forces are present, they have to be included in the usual way in the left side of Eq. (11). Physically, this type of PTF behavior can appear, in particular, for the case of a turbulent plasma, when the development of some instability can create a strong chaotic electrical field or irregular chaotic motion of one sort of particles with a prescribed nonMaxvellian distribution function. In such a turbulent plasma scattering with large transferring momenta can play a crucial role.

\section{REPRESENTATION IN MOMENTUM SPACE AND CONNECTION WITH THE FRACTIONAL DIFFERENTIATION APPROACH}

As mentioned, in general Eq. (11) cannot be written in terms of fractional differentiation. It confirms that the approach to anomalous diffusion based on the Fourier transfor- mation of the PTFs, in the form applied in this paper (see also Refs. 13 and 19) is a more general way for the problems under consideration.

However, for the purely power-type dependence of the functions $W(q) \equiv a / q^{\alpha}$ and $\widetilde{W}^{\prime}(q) \equiv b / q^{\beta}$, where $\alpha$ and $\beta$ are independent and satisfy the inequalities $5>\alpha>3$ and $5>\beta$ $>2$, Eq. (46) is appropriate and can be represented after inverse Fourier transformation in the following form (with the fractional derivatives):

$$
\frac{d f(\mathbf{p}, t)}{d t}=P_{0} \Delta^{v} f(\mathbf{p}, t)+P_{1} \Delta^{\lambda}\left(\lambda+\frac{\partial}{\partial \mathbf{p}} \mathbf{p}\right) f(\mathbf{p}, t),
$$

where $\nu \equiv(\alpha-3)(2>\nu>0) ; \lambda=\beta-5(2>\lambda>0)$. Here we introduced the fractional differentiation operator in the momentum space $\Delta^{\lambda} f(\mathbf{p}, t) \equiv \int d \mathbf{s} s^{2 \lambda} \exp (-i \mathbf{p s}) f(\mathbf{s}, t)$.

Let us consider now formally a specific particular model of anomalous diffusion, for which we assume a structure of the PTF $W(\mathbf{q}, \mathbf{p})$ with a rapid (say, exponential) decrease in the function $\tilde{W}^{\prime}(q)$. Therefore, the exponential function under the integrals in the coefficients $B(s)$ can be expanded, implying $B(s)=B_{0}$ [or $\beta=5$ and $B_{0} \equiv P_{1}$ in the notations of Eq. (46)]. At the same time the function $W(q) \equiv a / q^{\alpha}$ has a purely power-law dependence on $q$. The kinetic equation (49) then reads

$$
\frac{d f(\mathbf{s}, t)}{d t}=P_{0} s^{\alpha-3} f(\mathbf{s}, t)+B_{0} s_{i} \frac{\partial}{\partial s_{i}} f(\mathbf{s}, t)
$$

or in momentum space, according to Eq. (52),

$$
\frac{d f(\mathbf{p}, t)}{d t}=P_{0} \Delta^{\nu} f(\mathbf{p}, t)-B_{0} \frac{\partial}{\partial p_{i}}\left[p_{i} f(\mathbf{p}, t)\right] .
$$

Equation (54) is similar to the corresponding equation in Ref. 22 where a model of the Langevin equation with a constant friction frequency $\nu_{0} \equiv-B_{0}$ has been considered. In Ref. 23 a similar model with $\nu=3 / 2$ has been applied to estimate the fusion rate in a hot rarified plasma.

The stationary solution of Eq. (53) is

$$
f(s)=C^{\prime} \exp \left[-\frac{P_{0} s^{\nu}}{\nu B_{0}}\right] .
$$

The corresponding distribution in $p$-space is proportional to the Levy-type distribution $W\left(y, \alpha^{\prime}\right)$ $\equiv(2 y / \pi) \int_{0}^{\infty} d t t \sin (y t) \exp \left(-t^{\alpha^{\prime}}\right)$,

$$
\begin{aligned}
f(p) & =C^{\prime} \int d^{3} s \exp (-i \mathbf{p s}) \exp \left[-\frac{P_{0} s^{\nu}}{\nu B_{0}}\right] \\
& \equiv \frac{4 \pi C^{\prime}}{p} \int_{0}^{\infty} d s s \sin (p s) \exp \left[-\frac{P_{0} s^{\nu}}{\nu B_{0}}\right],
\end{aligned}
$$

with $y \equiv p\left(\nu B_{0} / P_{0}\right)^{1 / \nu}$ and $\alpha^{\prime} \equiv \nu$. As an example for the case $\nu=1$ we find $f(\mathbf{p})$,

$$
f(p)=\frac{8 \pi C^{\prime} P_{0}}{B_{0}\left[\left(p^{2}+4 P_{0}^{2} / B_{0}^{2}\right)\right]^{2}} .
$$

In the case $\nu=1$ the long tail of the distribution is proportional to $p^{-4}$ and the distribution $f(p)$ corresponds with the Cauchy-Lorentz distribution. Normalization of the distribu- 
tion $f(p)$ leads to the value $C^{\prime}=n /(2 \pi)^{3}$, where $n=N / V$ is the average density of particles undergoing diffusion in velocity space. A similar approach can be taken for other types of anomalous diffusion in velocity space.

\section{CONCLUSIONS}

In the present paper the problem of anomalous diffusion for plasmalike systems in momentum (velocity) space is rigorously analyzed. A new kinetic equation for anomalous diffusion in velocity space has been derived recently in Ref. 19, without suggesting any stationary equilibrium distribution function. We applied this equation to a system of charged particles with different masses to describe diffusion of heavy particles (ions and charged grains) in the surrounding light particles (electrons for the electron-ion plasma, electrons, and ions for dusty plasmas ${ }^{28}$ ). The distribution of the light particles can be non-Maxwellian, which is the cause of the appearance of long tails in the PTF. Conditions of convergence for the coefficients of the kinetic equation have been derived for a number of particular cases. It is found that a wide variety of anomalous processes in velocity space exists.

In general the Einstein relation for such a situation is not applicable because the stationary state may be far from equilibrium. For the case of normal diffusion the friction and diffusion coefficients have been found explicitly for the nonequilibrium case. For the equilibrium case the known Fokker-Planck equation in plasma is reproduced as a particular case.

\section{ACKNOWLEDGMENTS}

The authors are thankful to A. M. Ignatov and A. G. Zagorodny for the valuable discussions of some problems, reflected in this paper. S.A.T. would like to thank the Netherlands Organization for Scientific Research (NWO) for support of his investigations on the problems of stochastic transport in gases, liquids, and plasmas.
${ }^{1}$ A. S. Monin and A. M. Yaglom, Statistical Fluid Mechanics: Mechanics of Turbulence (MIT Press, Cambridge, 1975), Vol. II, p. 77.

${ }^{2}$ B. Rinn, P. Maass, and J.-P. Bouchaud, Phys. Rev. Lett. 84, 5403 (2000).

${ }^{3}$ R. Metzler and J. Klafter, Phys. Rep. 339, 1 (2000).

${ }^{4}$ H. Scher and E. W. Montroll, Phys. Rev. B 12, 2455 (1975).

${ }^{5}$ J. Klafter, M. F. Schlezinger, and G. Zumofen, Phys. Today 49(2), 33 (1996).

${ }^{6}$ A. Ott, J.-P. Bouchaud, D. Langevin, and W. Urbach, Phys. Rev. Lett. 65, $2201(1990)$.

${ }^{7}$ I. M. Sokolov, J. May, and A. Blumen, Phys. Rev. Lett. 79, 857 (1997).

${ }^{8}$ P. Gopikrishnan, V. Plerou, L. A. N. Amaral, M. Meyer, and H. E. Stanley, Phys. Rev. E 60, 5305 (1999).

${ }^{9}$ B. J. West, M. Bologna, and P. Grigolini, Physics of Fractal Operators (Springer-Verlag, New York, 2003), p. 194.

${ }^{10}$ H. C. Fogedby, Phys. Rev. Lett. 73, 2517 (1994).

${ }^{11}$ S. Jespersen, R. Metzler, and H. C. Fogedby, Phys. Rev. E 59, 2736 (1999).

${ }^{12}$ S. A. Trigger, G. J. F. van Heijst, and P. P. J. M. Schram, Physica A 347, 77 (2005).

${ }^{13}$ S. A. Trigger, G. J. F. van Heijst, and P. P. J. M. Schram, J. Phys.: Conf. Ser. 11, 37 (2005).

${ }^{14}$ S. A. Trigger, Phys. Lett. A 372, 1288 (2008).

${ }^{15}$ L. D. Landau, Zh. Eksp. Teor. Fiz. 7, 203 (1937).

${ }^{16}$ O. Klein, Ark. Mat., Astron, Fys, 16, 1 (1921).

${ }^{17}$ H. A. Kramers, Physica (Amsterdam) 7, 284 (1940).

${ }^{18}$ B. A. Carreras, D. Newman, V. E. Lynch, and P. H. Diamond, Plasma Phys. Rep. 22, 740 (1996); Phys. Plasmas 3, 2903 (1996); L. W. Owen, B. A. Carreras, R. Maingi, P. K. Mioduszewski, T. N. Carlstrom, and R. J. Groebner, Plasma Phys. Controlled Fusion 40, 717 (1998).

${ }^{19}$ S. A. Trigger, Phys. Lett. A 374, 134 (2009).

${ }^{20}$ E. Barkai, Phys. Rev. E 68, 055104(R) (2003).

${ }^{21}$ E. Barkai, J. Stat. Phys. 115, 1537 (2004).

${ }^{22}$ A. V. Chechkin, V. Yu. Gonchar, and M. Szydlowski, Phys. Plasmas 9, 78 (2002).

${ }^{23}$ W. Ebeling and M. Yu. Romanovsky, Contrib. Plasma Phys. 49, 479 (2009).

${ }^{24}$ J. L. Lebowitz and P. G. Bergmann, Ann. Phys. (N.Y.) 1, 1 (1957).

${ }^{25}$ N. G. van Kampen, Stochastic Processes in Physics and Chemistry (North-Holland, Amsterdam, 1992), p. 83.

${ }^{26}$ E. M. Lifshitz and L. P. Pitaevsky, Physical Kinetics (Nauka, Moscow, 1979), p. 116 (in Russian).

${ }^{27}$ S. I. Braginskii, The Problems of Plasma Theory (Gosatomizdat, Moscow, 1963), Vol. 1, p. 197 ( in Russian).

${ }^{28}$ V. E. Fortov, A. V. Ivlev, S. A. Khrapak, A. G. Khrapak, and G. E. Morfill, Phys. Rep. 421, 1 (2005). 